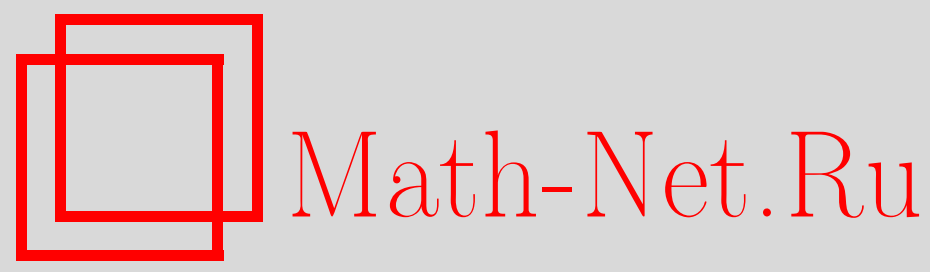

Ю. В. Бородина, П. А. Бородин, Синтез легкотестируемых схем в базисе Жегалкина при константных неисправностях типа 0 на выходах элементов, Дискрет. матем., 2010, том 22, выпуск 3, 127-133

DOI: https://doi.org/10.4213/dm1112

Использование Общероссийского математического портала Math-Net.Ru подразумевает, что вы прочитали и согласны с пользовательским соглашением http://www . mathnet.ru/rus/agreement

Параметры загрузки:

IP : 52.6 .47 .48

26 апреля 2023 г., $17: 40: 34$ 


\title{
Синтез легкотестируемых схем в базисе Жегалкина при константных неисправностях типа 0 на выходах элементов
}

\author{
() 2010 г. Ю. В. Бородина, П. А. Бородин
}

\begin{abstract}
Предложены методы синтеза легкотестируемых схем из функциональных элементов в базисе Жегалкина для произвольных булевых функций. В качестве неисправностей предполагаются константные неисправности типа 0 на выходах элементов. Доказано, что любую булеву функцию можно реализовать схемой, допускающей полный проверяющий тест длины 1.

Работа первого автора выполнена при поддержке Российского фонда фундаментальных исследований, проект 08-01-00863, и программы Президента Российской Федерации поддержки ведущих научных школ, проект НШ 4470.2008.1.

Работа второго автора выполнена при поддержке Российского фонда фундаментальных исследований, проект 08-01-00648.
\end{abstract}

Будем рассматривать схемы из функциональных элементов в базисе Жегалкина $\{\&, \oplus, 1,0\}$ $($ см. $[1,2])$ и в качестве неисправностей константные неисправности типа 0 на выходах конъюнкторов \& и сумматоров $\oplus$ (при переходе в неисправное состояние элемент выдает значение 0 независимо от входных данных).

Пусть $S$ - некоторая схема из функциональных элементов, реализующая булеву функцию $f(\tilde{x}), \tilde{x}=\left(x_{1}, x_{2}, \ldots, x_{n}\right)$.

Функция, реализуемая на выходе схемы при наличии в схеме неисправного элемента, называется функцией неисправности. Всякое множество $T$ входных наборов схемы $S$ называется полным проверяющим тестом для этой схемы, если для любой функции неисправности $g(\tilde{x})$, не равной тождественно $f(\tilde{x})$, в $T$ найдется хотя бы один такой набор $\tilde{\sigma}$, что $f(\tilde{\sigma}) \neq g(\tilde{\sigma})$ (см. [3, 4]). Число наборов, составляющих этот тест, называется длиной теста. При нахождении полных проверяющих тестов предполагается, что в неисправное состояние может переходить любое число элементов схемы. Если же в схеме предполагается не более одного неисправного элемента, говорят о единичных проверяющих тестах.

Задача синтеза легкотестируемых относительно единичных неисправностей схем становится корректной, если рассматривать нетривиальные неизбыточные схемы, то есть схемы, каждая из которых реализует отличную от константы функцию $f$ и при переходе любого одного элемента в неисправное состояние реализует функцию неисправности, отличную от $f$ (см. [4], гл. 4, §3).

Произвольный элемент схемы будем считать избыточным, если при переходе этого элемента в неисправное состояние реализуемая схемой функция не изменяется. Если некоторая схема избыточна, то удаляя из нее в произвольной последовательности избыточные элементы и, возможно, изменяя соединения оставшихся в схеме элементов между 


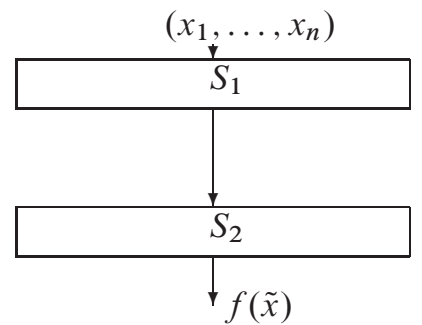

собой и с входами схемы, можно перейти от исходной схемы к некоторой неизбыточной схеме, реализующей ту же самую функцию, что и исходная схема.

Для единичных константных неисправностей произвольного типа (0 или 1) на выходах элементов в [5] доказано, что любую булеву функцию от $n$ переменных можно реализовать схемой в рассматриваемом базисе, допускающей единичный проверяющий тест длины не более $n+3$.

В случае единичных константных неисправностей типа 1 этот результат был уточнен в [6]: было доказано, что любую булеву функцию от $n$ переменных можно реализовать схемой в рассматриваемом базисе, допускающей единичный проверяющий тест длины 1.

Целью данной работы является получение точного значения (именно, 1) длины полного проверяющего теста для схем, реализующих произвольные булевы функции в базисе $\{\&, \oplus, 1,0\}$ в случае неисправностей типа 0 на выходах элементов (см. теорему 2).

Отметим, что теорема 2 не влечет никаких оценок длин тестов в случае константных неисправностей типа 1. Кроме того, конструкция схемы в теореме 2 не позволяет следить за сложностью этой схемы, и эта сложность, по-видимому, получается очень большой. Поэтому мы предваряем основной результат, теорему 2, более простым утверждением, в котором есть возможность оценить сложность схемы.

Теорема 1. Любую булеву функиию $f$ можно реализовать неизбыточной схемой из функцииональных элементов в базисе $\{\&, \oplus, 1,0\}$, допускающей в случае единичных неисправностей типа 0 на выходах элементов единичный проверяющий тест длины, не превосходящей 2. При этом сложность схемы (число ее элементов) равна суммарному числу умножений и сложений в полиноме Жегалкина функции $f$.

Доказательство. Пусть $f(\tilde{x})$ - произвольная булева функция, $\tilde{x}=\left(x_{1}, x_{2}, \ldots, x_{n}\right)$. Будем предполагать, что функция $f$ не является константой (константные функции являются базисными и реализуются без сумматоров и конъюнкторов) и существенно зависит от всех $n$ переменных. Это предположение не умаляет общности: если некоторая функция $f^{*}$ получается из $f$ изъятием фиктивных переменных и для $f^{*}$ утверждение теоремы справедливо, то это утверждение справедливо и для $f$.

Представим функцию $f(\tilde{x})$ полиномом Жегалкина:

$$
f\left(x_{1}, \ldots, x_{n}\right)=\sum_{m=1}^{n} \sum_{1 \leqslant i_{1}<\ldots<i_{m} \leqslant n} a_{i_{1}, \ldots, i_{m}} x_{i_{1}} \ldots x_{i_{m}} \oplus c,
$$

где суммы берутся по модулю $2, a_{i_{1}, \ldots, i_{m}}, c \in\{0,1\}$.

Схему $S$, реализующую функцию $f(\tilde{x})$, составим из двух подсхем $S_{1}$ и $S_{2}$ (см. рис. 1$)$.

Подсхема $S_{1}$ реализует все конъюнкции, входящие в полином Жегалкина функции $f(\tilde{x})$, цепями из конъюнкторов.

Подсхема $S_{2}$ представляет собой цепь из сумматоров и реализует сумму по модулю 2 выходных функций всех цепей подсхемы $S_{1}$. При этом на левый вход самого верхнего 
сумматора подается выход цепи, реализующей конъюнкцию $K$ минимальной длины (если $c=1$, то эта минимальная длина равна нулю, $K \equiv 1$, и на левый вход самого верхнего сумматора подается базисная функция 1; если таких конъюнкций в полиноме Жегалкина несколько, выберем любую из них).

Очевидно, что полученная схема $S$ реализует функцию $f(\tilde{x})$.

Докажем, что единичным проверяющим тестом для схемы $S$ будет множество $\left\{\tilde{\sigma}_{1}, \tilde{\sigma}_{2}\right\}$, где оба набора имеют длину $n$, набор $\tilde{\sigma}_{1}=(1, \ldots, 1)$, а набор $\tilde{\sigma}_{2}$ строится следующим образом: разряд, соответствующий переменной $x_{i}$, равен единице, если $x_{i}$ входит в конъюнкцию $K$ в качестве сомножителя, и нулю в ином случае. (При $f=x_{1} \& \ldots \& x_{n}$ имеем $\tilde{\sigma}_{1}=\tilde{\sigma}_{2}$.) В частности, если $K \equiv c \equiv 1$, то $\tilde{\sigma}_{2}=(0, \ldots, 0)$. Таким образом, на наборе $\tilde{\sigma}_{2}$ все конъюнкции полинома Жегалкина, кроме $K$, принимают значение 0 .

В подсхеме $S_{2}$ занумеруем все сумматоры числами $1,2, \ldots$, начиная с самого верхнего в цепи, на левый вход которого подается значение конъюнкции $K$.

Пусть все элементы схемы $S$ исправны. Тогда при подаче на входы схемы набора $\tilde{\sigma}_{1}$ на выходах всех конъюнкторов схемы (а следовательно, и на всех выходах подсхемы $S_{1}$ ) и сумматоров с четными номерами реализуются единицы, на выходах же сумматоров с нечетными номерами - нули. При подаче на входы схемы набора $\tilde{\sigma}_{2}$ цепь, реализующая конъюнкцию $K$, выдает единицу, на выходе остальных цепей подсхемы $S_{1}$ реализуются нули.

Пусть схема $S$ неисправна. Это означает, что ровно один элемент схемы перешел в неисправное состояние и выдает нуль при любых значениях, подаваемых на его входы.

Рассмотрим случай неисправности какого-либо конъюнктора подсхемы $S_{1}$. При переходе в неисправное состояние конъюнктора, являющегося выходным для подсхемы, на наборе $\tilde{\sigma}_{1}$ на вход какого-то сумматора подсхемы $S_{2}$ вместо единицы будет подан нуль, и значение на выходе схемы изменится с $f\left(\tilde{\sigma}_{1}\right)$ на противоположное, то есть неисправность будет обнаружена. Так как подсхема $S_{1}$ состоит из цепей из конъюнкторов, на входы которых на наборе $\tilde{\sigma}_{1}$ в исправном состоянии схемы подаются единицы, при переходе в неисправное состояние одного элемента цепи, не являющегося выходным, на левый вход следующего элемента будет подан нуль. Тогда мы получим нуль и на выходе всей цепи, а значит, значение на выходе всей схемы не будет равно $f\left(\tilde{\sigma}_{1}\right)$.

Неисправность сумматоров подсхемы $S_{2}$ обнаруживается при подаче на входы схемы набора $\tilde{\sigma}_{2}$. Действительно, в исправном состоянии схемы каждый сумматор на этом наборе выдает единицу, а при переходе любого сумматора в неисправное состояние значение на выходе схемы на этом наборе меняется на противоположное.

Теорема 1 доказана.

Теорема 2. Любую булеву функичю можно реализовать схемой из функциональных элементов в базисе $\{\&, \oplus, 1,0\}$, допускающей в случае константных неисправностей типа 0 на выходах элементов полный проверяющий тест длины 1.

Доказательство. Пусть $f(\tilde{x})$, где $\tilde{x}=\left(x_{1}, x_{2}, \ldots, x_{n}\right),-$ произвольная булева функция. Будем предполагать, что функция $f$ не является константой (константные функции являются базисными и реализуются без сумматоров и конъюнкторов) и существенно зависит от всех $n$ переменных. Это предположение не умаляет общности, так как если некоторая функция $f^{*}$ получается из $f$ изъятием фиктивных переменных и для $f^{*}$ утверждение теоремы справедливо, то это утверждение справедливо и для $f$.

Пусть $\tilde{\sigma}=\tilde{\sigma}(f)=\left(\sigma_{1}, \ldots, \sigma_{n}\right)$ - единичный набор функции $f$ (то есть $\left.f(\tilde{\sigma})=1\right)$, содержащий минимальное число единиц. Без ограничения общности можно считать, что $\tilde{\sigma}=(1, \ldots, 1,0, \ldots, 0)$ содержит $k$ единиц и $n-k$ нулей. 
Покажем, что функцию $f$ можно реализовать такой схемой $S$ в базисе $\{\&, \oplus, 1,0\}$, что переход в неисправное состояние любого числа элементов $S$ обнаружится на наборе $\tilde{\sigma}$.

Для обнаружения неисправности любого элемента на одном наборе $\tilde{\sigma}$ необходимо, чтобы при подаче набора $\tilde{\sigma}$ на вход схемы $S$ в исправном состоянии на выходе каждого ее элемента получалось значение 1. Для этого, в свою очередь, необходимо, чтобы одновременно выполнялись два следующих условия:

(a) на оба входа любого конъюнктора и на один вход любого сумматора подаются либо значения переменных $x_{1}, \ldots, x_{k}$, либо базисная функция 1 , либо выходы других элементов;

(б) на другой вход любого сумматора подается либо базисная функция 0 , либо значения переменных $x_{k+1}, \ldots, x_{n}$.

При этом выполнения условий (а) и (б) достаточно для того, чтобы набор б был полным проверяющим тестом схемы $S$. Пусть элемент $E$ схемы $S$ перешел в неисправное состояние. Если $E$ - выходной элемент схемы $S$, то неисправность будет обнаружена на наборе $\tilde{\sigma}$. Если же $E$ - промежуточный элемент схемы, то его выходное значение 0 будет подано на вход следующего за ним элемента $E^{\prime}$ (таких элементов может быть несколько). Если $E^{\prime}-$ конъюнктор, то на его выходе будет 0 независимо от значения, подаваемого на второй вход, а если $E^{\prime}-$ сумматор, то на второй его вход, согласно условию (б), подается нулевое значение одной из переменных $x_{k+1}, \ldots, x_{n}$, и на его выходе тоже будет 0 . На выходах следующих за $E^{\prime}$ элементов также получится 0 ; поскольку схема $S$ - граф без ориентированных циклов с единственным выходным элементом, значение 0 дойдет до выхода схемы $S$ и неисправность будет обнаружена $(f(\tilde{\sigma})=1)$.

Назовем $\tilde{\sigma}$-схемой любую схему в базисе $\{\&, \oplus, 0,1\}$, удовлетворяющую условиям (a) и (б). Также будем считать $\tilde{\sigma}$-схемами “пустые” (не содержащие конъюнкторов и сумматоров) схемы, реализующие константы.

Таким образом, для доказательства теоремы достаточно доказать следующее утверждение.

Предложение 1. Любую булеву функиию $f$ можно реализовать $\tilde{\sigma}(f)$-схемой.

Для доказательства этого предложения понадобится несколько вспомогательных утверждений. В нижеследующих леммах $1-4$ полагается, что $\tilde{\sigma}=(1, \ldots, 1,0, \ldots, 0)$ состоит из $k$ единиц и $n-k$ нулей.

Лемма 1. Если каждая из функичй $f_{1} u f_{2}$ реализуется б-схемой, то и функияия $f_{1} \& f_{2}$ реализуется бо-схемой.

Ясно, что достаточно подать выходы $\tilde{\sigma}$-схем для $f_{1}$ и $f_{2}$ на вход конъюнктора.

Лемма 2. Пусть функции $g_{1}\left(x_{k+1}, \ldots, x_{n}\right)$ u $g_{2}\left(x_{k+1}, \ldots, x_{n}\right)$ таковь, что для любой функиии $h$, которая реализуется $\tilde{\sigma}$-схемой, функции $h \oplus g_{1} u h \oplus g_{2}$ реализуются б-схемами. Тогда для любой функиии $f$, реализуемой $\tilde{\sigma}$-схемой, функция $f \oplus g_{1} g_{2}$ также реализуется бо-схемой.

Доказательство. Справедливы равенства

$$
\begin{aligned}
& \left(\left(f \oplus g_{1}\right)\left(f \oplus g_{2}\right) \oplus g_{1} \oplus g_{2}\right)\left(f\left(1 \oplus g_{1}\right) \oplus g_{1}\right)\left(f\left(1 \oplus g_{2}\right) \oplus g_{2}\right) \\
& \quad=\left(f \oplus f g_{1} \oplus f g_{2} \oplus g_{1} g_{2} \oplus g_{1} \oplus g_{2}\right)\left(f \oplus g_{1}(f \oplus 1)\right)\left(f \oplus g_{2}(f \oplus 1)\right) \\
& \quad=\left(f \oplus g_{1}(f \oplus 1) \oplus g_{2}(f \oplus 1) \oplus g_{1} g_{2}\right)\left(f \oplus g_{1} g_{2}(f \oplus 1)\right) \\
& \quad=f \oplus g_{1} g_{2} f \oplus g_{1} g_{2}(f \oplus 1)=f \oplus g_{1} g_{2} .
\end{aligned}
$$


Первая формула в этом равенстве дает способ реализации функции $f \oplus g_{1} g_{2} \tilde{\sigma}$-схемой: функции $f \oplus g_{1}, f \oplus g_{2}, 1 \oplus g_{1}, 1 \oplus g_{2}$ реализуются $\tilde{\sigma}$-схемами по условию леммы, функции $\left(f \oplus g_{1}\right)\left(f \oplus g_{2}\right), f\left(1 \oplus g_{1}\right), f\left(1 \oplus g_{2}\right)$ - по лемме 1 , функции в каждой из трех скобок - по условию леммы 2, а их произведение - по лемме 1.

Лемма 3. Пусть функциюю $f\left(x_{1}, \ldots, x_{n}\right)$ можно реализовать б̃-схемой. Тогда для любой функции $g\left(x_{k+1}, \ldots, x_{n}\right), g(0, \ldots, 0)=0$, функцию $f \oplus g$ также можно реализовать $\tilde{\sigma}$-схемой.

Доказательство. Достаточно доказать это утверждение для мономов $x_{j_{1}} \& \ldots \& x_{j_{m}}$, где $j_{i} \in\{k+1, \ldots, n\}, i=1, \ldots, m$. Для любой функции $h$, которая реализуется $\tilde{\sigma}$-схемой, функции $h \oplus x_{j_{i}}$, очевидно, реализуются $\tilde{\sigma}$-схемами. Тогда из леммы 2 следует, что и функцию $f \oplus x_{j_{1}} \& \ldots \& x_{j_{m}}$ можно реализовать $\tilde{\sigma}$-схемой.

Лемма 4. Пусть каждая из функиий $f_{1}$ и $f_{2}$ реализуется $\tilde{\sigma}$-схемой. Тогда для любой функции $g\left(x_{k+1}, \ldots, x_{n}\right), g(0, \ldots, 0)=0$, функция $f_{1} f_{2} \oplus g f_{1} \oplus g f_{2} \oplus g f_{1} f_{2}$ реализуется $\tilde{\sigma}$-схемой.

Доказательство. Справедливы равенства

$$
\begin{array}{r}
\left(f_{1}\left(f_{2} \oplus g\right) \oplus g\right)\left(f_{2}\left(f_{1} \oplus g\right) \oplus g\right) \oplus g=\left(f_{1} f_{2} \oplus g\left(f_{1} \oplus 1\right)\right)\left(f_{1} f_{2} \oplus g\left(f_{2} \oplus 1\right)\right) \oplus g \\
=f_{1} f_{2} \oplus g\left(f_{1} \oplus 1\right)\left(f_{2} \oplus 1\right) \oplus g=f_{1} f_{2} \oplus g f_{1} f_{2} \oplus g f_{1} \oplus g f_{2} .
\end{array}
$$

Первая формула в этом равенстве дает способ реализации $\tilde{\sigma}$-схемой функции $f_{1} f_{2} \oplus g f_{1} \oplus g f_{2} \oplus g f_{1} f_{2}$ : функции $f_{1} \oplus g, f_{2} \oplus g$ реализуются $\tilde{\sigma}$-схемами по лемме 3 , функции $f_{1}\left(f_{2} \oplus g\right), f_{2}\left(f_{1} \oplus g\right)$ - по лемме 1 , функции в скобках - по лемме 3 , их произведение - по лемме 1 , а сумма их произведения и функции $g-$ по лемме 3 .

Вернемся к доказательству предложения 1. Будем доказывать его индукцией по $n$.

При $n=1$ предложение 1 достаточно очевидно: функции $1, \bar{x}_{1}=1 \oplus x_{1}$, $x_{1}$ реализуются $\tilde{\sigma}$-схемами. Для 1 и $\bar{x}_{1}$ имеем $k=0$, для $x_{1}$ имеем $k=1$; функции 1 и $x_{1}$ реализуются без конъюнкторов и сумматоров, схема для $\bar{x}_{1}$ содержит один сумматор, на один вход которого подается значение переменной $x_{1}$, а на другой вход подается базисная функция 1.

Предположим, что любая функция $g$ от $n-1$ переменных, отличная от тождественного нуля, реализуется $\tilde{\sigma}(g)$-схемой (набор $\tilde{\sigma}(g)$ и число $k-$ свои для каждой функции $g$ ). Пусть $f\left(x_{1}, \ldots, x_{n}\right), n \geqslant 2,-$ произвольная функция, отличная от констант, $\tilde{\sigma}=\tilde{\sigma}(f)=(1, \ldots, 1,0, \ldots, 0), k, 0 \leqslant k \leqslant n,-$ число единиц.

Если $k=0$, то $f(\tilde{x})=1 \oplus g\left(x_{1}, \ldots, x_{n}\right)$, где $g(0, \ldots, 0)=0$. Константа 1 реализуется $\tilde{\sigma}$-схемой с $k=0$, поэтому функция $f$ реализуется $\tilde{\sigma}$-схемой по лемме 3 .

Если $k=n$, то $f(\tilde{x})=x_{1} \& \ldots \& x_{n}$, и $\tilde{\sigma}$-схемой для $f$ является цепь из конъюнкторов (см. рис. 2).

Если $k=1$, то

$$
f(\tilde{x})=x_{1}\left(1 \oplus g_{1}\left(x_{2}, \ldots, x_{n}\right)\right) \oplus g_{2}\left(x_{2}, \ldots, x_{n}\right),
$$

где $g_{1}(0, \ldots, 0)=g_{2}(0, \ldots, 0)=0$. Функция $1 \oplus g_{1}\left(x_{2}, \ldots, x_{n}\right)$ реализуется $\tilde{\sigma}$-схемой без участия $x_{1}$ по лемме 3 , функция $x_{1}\left(1 \oplus g_{1}\right)$ - по лемме 1 , наконец, функция $f=x_{1}\left(1 \oplus g_{1}\right) \oplus g_{2}-$ по лемме 3 .

Пусть теперь $2 \leqslant k \leqslant n-1$. Рассмотрим две функции, пусть $I_{0}\left(x_{1}, \ldots, x_{n}\right)-$ индикаторная функция тех наборов, для которых либо $x_{k+1}=x_{k+2}=\ldots=x_{n}=0$, 


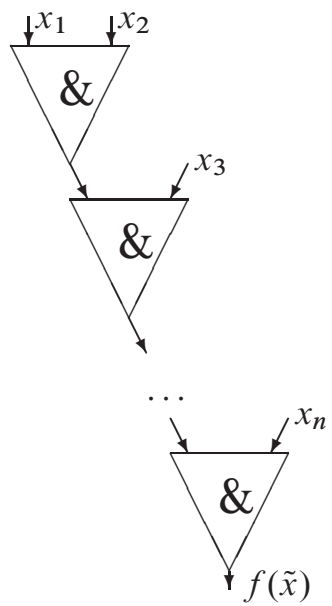

Pис. 2.

либо $x_{1}=0$, и $I_{1}\left(x_{1}, \ldots, x_{n}\right)$ - индикаторная функция тех наборов, для которых либо $x_{k+1}=x_{k+2}=\ldots=x_{n}=0$, либо $x_{1}=1$. Положим $f_{0}=f \& I_{0}, f_{1}=f \& I_{1}$, $g\left(x_{k+1}, \ldots, x_{n}\right)=x_{k+1} \vee x_{k+2} \vee \ldots \vee x_{n}$.

Покажем, что

$$
f=f_{0} f_{1} \oplus g f_{0} \oplus g f_{1} \oplus g f_{0} f_{1} .
$$

Действительно, при $x_{k+1}=x_{k+2}=\ldots=x_{n}=0$ справедливы равенства $g=0$, $I_{0}=1, I_{1}=1$ и $f_{0} f_{1} \oplus g f_{0} \oplus g f_{1} \oplus g f_{0} f_{1}=f_{0} \& f_{1}=f I_{0} \& f I_{1}=f$. Если же значение хотя бы одной из переменных $x_{k+1}, \ldots, x_{n}$ отлично от 0 , то $g=1, I_{0}=1 \oplus x_{1}, I_{1}=x_{1}$ и $f_{0} f_{1} \oplus g f_{0} \oplus g f_{1} \oplus g f_{0} f_{1}=f_{0} \oplus f_{1}=f I_{0} \oplus f I_{1}=f\left(1 \oplus x_{1}\right) \oplus f x_{1}=f$. В силу формулы предложения 1 и леммы 4 достаточно доказать, что каждая из функций $f_{0}$ и $f_{1}$ реализуется $\tilde{\sigma}$-схемой.

Для функции $f_{0}$ набор $\tilde{\sigma}=(1, \ldots, 1,0, \ldots, 0)$ с $k$ единицами и $n-k$ нулями попрежнему является единичным набором с наименьшим числом единиц. Рассмотрим функции

$$
\begin{aligned}
f_{01}\left(x_{2}, \ldots, x_{n}\right) & =f_{0}\left(0, x_{2}, \ldots, x_{n}\right) \vee f_{0}\left(1, x_{2}, \ldots, x_{n}\right), \\
f_{02}\left(x_{1}, x_{3}, \ldots, x_{n}\right) & =f_{0}\left(x_{1}, 0, x_{3}, \ldots, x_{n}\right) \vee f_{0}\left(x_{1}, 1, x_{3}, \ldots, x_{n}\right) .
\end{aligned}
$$

Покажем, что

$$
f_{0}\left(x_{1}, \ldots, x_{n}\right)=f_{01}\left(x_{2}, \ldots, x_{n}\right) \& f_{02}\left(x_{1}, x_{3}, \ldots, x_{n}\right) \text {. }
$$

Если для какого-то набора $\tilde{\alpha}=\left(\alpha_{1}, \ldots, \alpha_{n}\right)$ справедливо равенство $f_{0}(\tilde{\alpha})=1$, то, как нетрудно видеть, $f_{01}\left(\alpha_{2}, \ldots, \alpha_{n}\right)=1$ и $f_{02}\left(\alpha_{1}, \alpha_{3}, \ldots, \alpha_{n}\right)=1$, так что формула (2) верна для набора $\tilde{\alpha}$.

Пусть теперь для набора $\tilde{\alpha}=\left(\alpha_{1}, \ldots, \alpha_{n}\right)$ справедливо равенство $f_{0}(\tilde{\alpha})=0$. Если $\alpha_{1}=1$ и $\alpha_{k+1}=\ldots=\alpha_{n}=0$, то среди $\alpha_{2}, \ldots, \alpha_{k}$ есть хотя бы один нуль и

$$
f_{01}\left(\alpha_{2}, \ldots, \alpha_{n}\right)=f_{0}\left(0, \alpha_{2}, \ldots, \alpha_{k}, 0, \ldots, 0\right) \vee f_{0}\left(1, \alpha_{2}, \ldots, \alpha_{k}, 0, \ldots, 0\right)=0 .
$$

Если же $\alpha_{1}=1$ и среди $\alpha_{k+1}, \ldots, \alpha_{n}$ есть хотя бы одна единица, то

$$
f_{02}\left(\alpha_{1}, \alpha_{3}, \ldots, \alpha_{n}\right)=f_{0}\left(1,0, \alpha_{3}, \ldots, \alpha_{n}\right) \vee f_{0}\left(1,1, \alpha_{3}, \ldots, \alpha_{n}\right)=0 \vee 0=0
$$


(поскольку $I_{0}\left(1, x_{2}, \ldots, x_{k}, \alpha_{k+1}, \ldots, \alpha_{n}\right)=0$, см. определение функции $\left.I_{0}(\tilde{x})\right)$.

Если $\alpha_{1}=0$ и $\alpha_{k+1}=\ldots=\alpha_{n}=0$, то

$$
f_{02}\left(\alpha_{1}, \alpha_{3}, \ldots, \alpha_{n}\right)=f_{0}\left(0,0, \alpha_{3}, \ldots, \alpha_{k}, 0, \ldots, 0\right) \vee f_{0}\left(0,1, \alpha_{3}, \ldots, \alpha_{k}, 0, \ldots, 0\right)=0 \text {. }
$$

Если же $\alpha_{1}=0$ и среди $\alpha_{k+1}, \ldots, \alpha_{n}$ есть хотя бы одна единица, то

$$
f_{01}\left(\alpha_{2}, \ldots, \alpha_{n}\right)=f_{0}\left(\alpha_{1}, \ldots, \alpha_{n}\right) \vee f_{0}\left(1, \alpha_{2}, \ldots, \alpha_{n}\right)=0 \vee 0=0
$$

$\left(f_{0}(\tilde{\alpha})=0\right.$ по предположению, а $f_{0}\left(1, \alpha_{2}, \ldots, \alpha_{n}\right)=0$ по определению $\left.f_{0}\right)$.

Таким образом, во всех случаях получается либо $f_{01}(\tilde{\alpha})=0$, либо $f_{02}(\tilde{\alpha})=0$, так что формула (2) верна.

Для функций $f_{01}\left(x_{2}, \ldots, x_{n}\right)$ и $f_{02}\left(x_{1}, x_{3}, \ldots, x_{n}\right)$ набор $(1, \ldots, 1,0, \ldots, 0)$ с $k-1$ единицами и $n-k$ нулями является единичным набором с наименьшим числом единиц, и по предположению индукции эти функции реализуются $\tilde{\sigma}$-схемами без участия переменных $x_{1}$ и $x_{2}$ соответственно.

Аналогично доказывается, что

$$
f_{1}\left(x_{1}, \ldots, x_{n}\right)=f_{11}\left(x_{2}, \ldots, x_{n}\right) \& f_{12}\left(x_{1}, x_{3}, \ldots, x_{n}\right),
$$

где

$$
\begin{aligned}
f_{11}\left(x_{2}, \ldots, x_{n}\right) & =f_{1}\left(0, x_{2}, \ldots, x_{n}\right) \vee f_{1}\left(1, x_{2}, \ldots, x_{n}\right), \\
f_{12}\left(x_{1}, x_{3}, \ldots, x_{n}\right) & =f_{1}\left(x_{1}, 0, x_{3}, \ldots, x_{n}\right) \vee f_{1}\left(x_{1}, 1, x_{3}, \ldots, x_{n}\right) .
\end{aligned}
$$

Для функций $f_{11}\left(x_{2}, x_{3}, \ldots, x_{n}\right)$ и $f_{12}\left(x_{1}, x_{3}, \ldots, x_{n}\right)$ набор $(1, \ldots, 1,0, \ldots, 0)$ с $k-1$ единицей и $n-k$ нулями является набором с наименьшим числом единиц, и по предположению индукции эти функции реализуются $\tilde{\sigma}$-схемами без участия переменных $x_{1}$ и $x_{2}$ соответственно.

В силу формул (2), (3) и леммы 1 функции $f_{0}$ и $f_{1}$ реализуются $\tilde{\sigma}$-схемами.

Предложение 1 и теорема 2 доказаны.

\section{Список литературы}

1. Лупанов О. Б., Асимптотические оценки сложности управляющих систем. МГУ, Москва, 1984.

2. Яблонский С. В., Введение в дискретную математику. Высшая школа, Москва, 2002.

3. Яблонский С. В., Некоторые вопросы надежности и контроля управляющих систем. Матем. вопросы кибернетики (1988) 1, 5-25.

4. Редькин Н. П., Надежность и диагностика схем. МГУ, Москва, 1992.

5. Reddy S. M., Easily testable realization for logic functions. IEEE Trans. Computers (1972) 21, 124-141.

6. Бородина Ю. В., О схемах, допускающих единичные тесты длины 1 при константных неисправностях на выходах элементов. Вестник Московского университета. Сер. 1, матем. механ. (2008), № 5, 49-52.

Статья поступила 23.01.2009. 